\title{
Stress relaxation of constructions elements
}

\author{
Evgeniy Larionov, ${ }^{*}$ Evgeniy Zveryaev ${ }^{2}$ \\ ${ }^{1}$ Moscow state university of civil engineering, Yaroslavskoye shosse, 26, Moscow, Russia, 129337 \\ ${ }^{2}$ Keldysh Institute of Applied Mathematics, Miusskaya sq. 4, Moscow, 125047, Russia
}

\begin{abstract}
A relaxation of stress in the elements of constructions is considered and an approach is proposed for solution of corresponding problems. It is notable that this approach is based on a modification Boltzmann's principle superposition of fraction creep deformations. This modification reduces the noted problems to solution of linear relative to socalled structural stress integral equations. Next a desired stress is defined by solution of algebraic equations. It should be underline that a material (concrete, steel, graph) of elements a considered as a union of its fractions with statistical disturbed strengths. This ascending to Weibull conception [1] permits to modify Boltzmann's principle superposition [2]. As a result this principle is applicable when a dependence on deformations from the stresses is nonlinear [3-7].
\end{abstract}

According to Berg [6] and Gvozdev [9] an increasing on cross-section loading $N(\tau)$ implies destruction a certain fractions of the element. Here $\tau$ is a current temp.

In consequence of this destruction an initial area $A$ of cross-section is decreased until $A(\tau)<A$. The area $A(\tau)$ represents a cross-section of all entire at instant $\tau$ fractions of the constructive element. $A$ connected with the structural damages of the element value

$$
\sigma_{c}(\tau)=\frac{N(\tau)}{A(\tau)}
$$

is called the structural stress. Since

$$
\sigma_{c}(\tau)=\frac{N(\tau)}{A}
$$

is the normal stress then according to the relations (1) and (2) we have

$$
\sigma_{c}(\tau)=\frac{A}{A(\tau)} \sigma(\tau)=S^{0}(\tau) \sigma(t)
$$

The function $S^{0}(\tau)$ describs the destruction of a certain part of element and is called the function of non-linearity of stress.

As $\sigma(\tau)=$ const $; \tau \in\left[t_{0}, t\right]$ we obtain the creep deformation

\footnotetext{
* Corresponding author: larionov.ea@gmail.com
} 


$$
\varepsilon_{c}\left(t_{0}, t\right)=C_{0}(t, t) \sigma(t)
$$

and the complete deformation is

$$
\varepsilon\left(t, t_{0}\right)=\frac{\sigma(t)}{E(t)}+C_{0}\left(t, t_{0}\right) \sigma(t)
$$

Here $\frac{\sigma(t)}{E(t)}$ is the instantaneous deformation $\varepsilon_{m}(t) ; C_{0}\left(t, t_{0}\right)$ is the measure of the simple creep; $E(t)$ is the elasticity module.

The increment $\Delta \sigma(t)=\sigma(t)-\sigma\left(t_{0}\right)$ of stress implies the creep deformation $\Delta \varepsilon_{c}\left(t, t_{0}\right)$. In virtue of the Boltzmann's principle of superposition the fraction increment $\Delta \varepsilon_{c}\left(t, \tau_{i}\right)$ is defined only by the value and duration $\Delta \sigma\left(\tau_{i}\right)$, and is independent on the rest increments $\Delta \sigma\left(\tau_{j}\right) ; j \neq i$.

Consequently

$$
\begin{aligned}
& \Delta \varepsilon_{c}\left(t, \tau_{i}\right)=C_{0}\left(t, \tau_{i}\right) \Delta \sigma\left(\tau_{i}\right) \\
& \Delta \varepsilon_{c}\left(t, t_{0}\right)=\sum_{i=1}^{n} C_{0}\left(t, \tau_{i}\right) \Delta \sigma\left(\tau_{i}\right) \\
& \Delta \varepsilon_{c}\left(t, t_{0}\right)=\int_{t_{0}}^{t} C_{0}(t, \tau) d \sigma(\tau)
\end{aligned}
$$

and integrating by parts, we have

$$
\Delta \varepsilon_{c}\left(t, t_{0}\right)=C_{0}(t, t) \sigma(t)-C_{0}\left(t, t_{0}\right) \sigma\left(t_{0}\right)-\int_{t_{0}}^{t} \sigma(\tau) \frac{\partial C_{0}(t, \tau)}{\partial \tau} d t
$$

Then, according to the equalities

$$
\begin{gathered}
\varepsilon\left(t, t_{0}\right)=\frac{\sigma(t)}{E(t)}+\varepsilon_{c}\left(t, t_{0}\right) \\
\varepsilon_{c}\left(t, t_{0}\right)=C_{0}\left(t, t_{0}\right) \sigma\left(t_{0}\right)+\Delta \varepsilon_{c}\left(t, t_{0}\right) \\
C_{0}(t, \tau)=\frac{1}{E(\tau)}-\frac{1}{E(t)}+C(t, \tau)
\end{gathered}
$$

we obtain the linear rheological state equation

$$
\varepsilon\left(t, t_{0}\right)=\frac{\sigma(t)}{E(t)}+C(t, t) \sigma(t)-\int_{t_{0}}^{t} \sigma(\tau) \frac{\partial}{\partial \tau} \frac{1}{E(\tau)} d \tau-\int_{t_{0}}^{t} \sigma(\tau) \frac{\partial C(t, \tau)}{\partial \tau} d \tau
$$

In the equation (13) the value $C(t, t) \sigma(t)$ represents the so-called quick creep deformation [10].

In consequence of the destruction of a certain fractions of the element and the next redistribution of the loading $N(\tau)$ every increment $\Delta \sigma\left(\tau_{k}\right)$ intensifies the action of the previous increment $\Delta \sigma\left(\tau_{i}\right) ;(i<k)$. This means the dependence of fraction creep deformation $\Delta \varepsilon_{c}\left(t, \tau_{i}\right)$ also on all next increments of the stress $\sigma(\tau)$ and therefore the superposition of $\Delta \varepsilon_{c}\left(t, \tau_{i}\right)$ relatively $\Delta \sigma\left(\tau_{i}\right)$ is impossible.

Non we show that this superposition is realized relatively $\Delta \sigma_{c}\left(\tau_{i}\right)=S^{0}\left(\tau_{i}\right) \Delta \sigma\left(\tau_{i}\right)$, of the fraction increments of the structural stress $\sigma_{c}(\tau)$. 
An interdependence on $\varepsilon_{c}\left(t, \tau_{i}\right)$ take place for an ideal element when all the fractions have the same strengths $R(\tau)$ and consequently the retribution of $N(\tau)$ is excluded. Therefore parallel with the given element we consider the geometrically identical to it ideal element with the same parameters $E(\tau)$ and $C(t, \tau)$. The ideal element under the axial loading $N_{c}(\tau)=\frac{A}{A(\tau)} N(\tau)=S^{0}(\tau) N(\tau)$ tests the stress $\sigma_{c}(\tau)$ and the increment $\Delta \sigma_{c}\left(\tau_{i}\right)$ generate $\Delta \varepsilon_{c}\left(t, \tau_{i}\right)$.

Thus we have

$$
\begin{gathered}
\Delta \varepsilon_{c}\left(t, \tau_{i}\right)=C_{0}\left(t, \tau_{i}\right) \Delta \sigma_{c}\left(\tau_{i}\right) \\
\Delta \varepsilon_{c}\left(t, t_{0}\right)=\sum_{i=1}^{n} C_{0}\left(t, \tau_{i}\right) \Delta \sigma_{c}\left(\tau_{i}\right) \\
\Delta \varepsilon_{c}\left(t, t_{0}\right)=\int_{t_{0}}^{t} C_{0}(t, \tau) d \sigma_{c}(\tau) \\
\Delta \varepsilon_{c}\left(t, t_{0}\right)=C(t, t) \sigma_{c}(t)-C\left(t, t_{0}\right) \sigma_{c}(t)-\int_{t_{0}}^{t} \sigma_{c}(\tau) \frac{\partial}{\partial \tau}\left[\frac{1}{E_{(\tau)}}+C(t, \tau)\right] d \tau \\
\varepsilon_{c}\left(t, t_{0}\right)=C(t, t) \sigma_{c}(t)-\int_{t_{0}}^{t} \sigma_{c}(\tau) \frac{\partial}{\partial \tau}\left[\frac{1}{E_{(\tau)}}+C(t, \tau)\right] d \tau
\end{gathered}
$$

According to equation (18) and $\sigma_{c}(\tau)=S^{0}(\tau) \sigma(\tau)$ we obtain the nonlinear rheological state equation

$$
\varepsilon\left(t, t_{0}\right)=S^{0}(t) \sigma(t)\left[\frac{1}{E(t)}+C(t, t)-\int_{t_{0}}^{t} \frac{S^{0}(\tau) \sigma(\tau)}{S^{0}(t) \sigma(t)} \frac{\partial}{\partial \tau}\left[\frac{1}{E(\tau)}+C(t, \tau)\right] d \tau\right.
$$

We can present an alternate vision of the deduction for nonlinear rheological equation (19). Consider to this end the part $V_{t}$ of the constructive element consisting from the union of all the remaining entire in the interval $\left[t_{0}, t\right]$ fractions. The loading $N(\tau)$ generates on its cross-section (with the area $A(t))$ same structural stress $\sigma_{c}(\tau)$ that on cross-section of the considered element (with the area $A(\tau)$ ).

The element and its part $V_{t}$ have same fraction creep deformations and the absence of one in the interval $\left[t_{0}, t\right]$ of the redistribution of the loading $N_{e}(\tau)=\frac{A(t)}{A} N(\tau)$ implies their interindepency. Then, for $V_{t}$ we have the relations (14)-(18) and consequently again obtain the rheological equation (19).

Evidently linear equation (13) contradicts to the knowns non-linear diagrams $\ll \sigma-\varepsilon »$.

Note that according to A.A. Gvozdev a non-linear part of $\langle\sigma-\varepsilon »$ is generated by quick creep at the momentum of loading [19]. In addition he assumes the linearity of the dependence $\varepsilon_{m}(\tau)$ on $\sigma(\tau)$. By A.A. Gvozdev the creep deformation is the composition of the linear component $\varepsilon_{c e}\left(t, t_{0}\right)=\int_{t_{0}}^{t} C^{*}(t, \tau) d \sigma(\tau)$ and the non-linear component 
$\varepsilon_{c n}\left(t, t_{0}\right)=\int_{t_{0}}^{t} L(t, \tau, \sigma) d \sigma(\tau)$, generated because of the structural damages. This conception implies a necessity of the choice of the functions $C^{*}$ and $L$ corresponding to the diagram $« \sigma-\varepsilon »$. We note that this case reduce to the considerable inconvenience for the applications [12].

In connection with diagram $\langle\sigma-\varepsilon »$ V.M. Bondarenko affirms that the dependence $\varepsilon_{m}(t)$ from $\sigma(t)$ also is non-linear and is described by the relation $\varepsilon_{m}(t)=S_{m}[\sigma(t)] / E(t)$ [13]. Since the instantaneous and creep deformations generated by the same stress $\sigma_{c}(\tau)$ their non-linear functions $S_{m}[\sigma(\tau)]$ and $S_{c}[\sigma(\tau)]$ coincide $S_{m}[\sigma(\tau)]=S_{c}[\sigma(\tau)]=A / A(\tau)[3,4]$.

In [14-16] the non-linear rheological equation is presented in the following form

$$
\varepsilon(t)=\varepsilon_{m}(t)-\int_{t_{0}}^{t} \sigma(\tau) \frac{\partial C(t, \tau)}{\partial \tau} d \tau
$$

This means that it is assumed (along with $C(t, t)=0$ and $E(\tau)=$ const) the possibility of the superposition $\Delta \varepsilon_{c}\left(t, \tau_{i}\right)$ relative to $\Delta \sigma\left(\tau_{i}\right)$. However (as is shown in section 2) such superposition takes place only in the linear creep theory.

Remark 1. The adequate to the physical essence of the creep phenomenon the nonlinear rheological equations are deduced only on basis of the corresponding modification Boltzmann's principle superposition.

Papiers [14-16] are useful for an analysis of the applied in our actual norms creep theory. In addition they are connected with the problem of correspondence of these norms and Eurocod.

Remark 2. A creep phenomenon (increment of $\varepsilon(t)$ under $\sigma(\tau)=\sigma\left(t_{0}\right) ; \tau \in\left[t_{0}, t\right]$ ) is the consequence of strength distribution on cross-section of the element. In fact a degradation of $R(\tau)$ implies an increment $\sigma_{c}(\tau)=\frac{A}{A(\tau)} \sigma\left(t_{0}\right)$ and therefore an increment of $\varepsilon(t)$.

Remark 3. In the course of taking down of the loading we have $S^{0}[\sigma(\tau)]=A / A(\tau)=$ const, hence, the dependence $\varepsilon(\tau)$ on $\sigma(\tau)$ is linear, that is observed on diagram $« \sigma-\varepsilon »$.

According to the equation (19) we have

$$
\begin{gathered}
\varepsilon\left(t, t_{0}\right)=\frac{\sigma_{c}(t)}{E(t)}+C(t, t) \sigma_{c}(t)-\int_{t_{0}}^{t} \sigma_{c}(\tau) \frac{\partial C_{0}}{\partial \tau}(t, \tau) d \tau \\
\sigma_{c}(t)=\hat{\sigma}_{c}(t)+\lambda(t) \int_{t_{0}}^{t} \sigma_{c}(\tau) \frac{\partial C_{0}(t, \tau)}{\partial \tau} d \tau
\end{gathered}
$$

Here $\hat{\sigma}(t)=\frac{\varepsilon\left(t, t_{0}\right) E(t)}{1+E(t) C(t, t)} ; \lambda(t)=\frac{E(t)}{1+E(t) C(t, t)}$ is the elastoplastic module of deformations. Solving the linear integral in equation (22) (par example by the iteration method) we come to an equation $S^{0}[\sigma(t)]=\sigma_{c}^{*}(t)$, where $\sigma_{c}^{*}(t)$ is the solution of equation (22). 
In applications the function $S^{0}(\tau)$ is represented usually in form

$$
S^{0}[\sigma(t)]=1+V \cdot\left[\frac{\sigma(t)}{R(t)}\right]^{m} \text { or } S^{0}[\sigma(t)]=a[\sigma(t)]^{b}
$$

where $V, m, a, b$ are the experimental parameters [17]. Then, we find $\sigma(t)$ as a solution of equation

$$
[\sigma(t)]^{m+1}+\frac{[R(t)]^{m}}{V} \sigma(t)-\frac{[R(t)]^{m}}{V} \sigma_{c}^{*}(t)=0 \text { or } a[\sigma(\tau)]^{b+1}=\sigma_{c}^{*}(t)
$$

If $C(t, \tau)=C(0, \infty)\left[1-\beta e^{-\gamma(t-\tau)}\right]$ the stress $\sigma(t)$ is defined by solution of corresponding to (19) linear differential eqnation.

As the loading $N(\tau)=N\left(t_{0}\right)$ at $\tau \geq t_{0}$ we have $S^{0}[\sigma(t)]=S^{0} \sigma\left(t_{0}\right)$ and therefore $\sigma(t)=\sigma_{c}^{*}(t) / S^{0}\left[\sigma\left(t_{0}\right)\right]$. This case take place when $\varepsilon\left(\tau, t_{0}\right)=\varepsilon\left(t_{0}\right)$ at $\tau \geq t_{0}$.

Let us illustrate the simple example of the stress relaxation in the concrete. Suppose that a reinforced concrete rod tests with the axial loading

$$
N(\tau)=\left\{\begin{array}{l}
N ; 2 k t \leq \tau<(2 k+1) T \\
N_{0} ;(2 k+1) T \leq \tau<2(k+1) T
\end{array}\right.
$$

where $T>0 ; N_{0}<N ; k=0,1,2, \ldots \ldots, n$.

The effort $N$ on the concrete part of the cross-section and $N_{0}$ generates the stresses

$$
\sigma_{1}=\frac{N}{A_{c}(1+m \mu)} \text { and } \sigma_{2}=\frac{N_{0}}{A_{c}(1+m \mu)}
$$

at the moment $t_{1}=2 k t$ and $t_{2}=(2 k+1) T$ respectively. Here $m=E_{s} / E_{c} ; \mu=A_{s} / A_{c}$; $E_{c}\left(E_{s}\right)$ is the elasticity module of the concrete (steel); $A_{c}\left(A_{s}\right)$ is the area of the crosssection of the concrete (steel).

Let $A_{1}$ be the corresponding to $\sigma_{1}$ area of the cross-section for all entire fractions. Taking into account that $A(\tau)=$ const on increasing loading and $\sigma_{2}<\sigma_{1}$ we obtain

$$
S^{0}(\tau)=1+V \cdot\left[\frac{\sigma_{1}}{R}\right]^{m} ; \tau \geq 0
$$

In given by relation (23) the intervals of temps we have

$$
\sigma(t)=\sigma_{i}+\lambda \int_{t_{i}}^{t} \sigma(\tau) e^{-\gamma(t-\tau)} d \tau ; i=1,2 ;
$$

Here

$$
\lambda=\frac{E_{s} \gamma \mu \beta C_{o}}{1+m \mu} ; t_{1}=2 k t ; t_{2}=(2 k+1) T ; k=0,1,2, \ldots \ldots ., n
$$

The equations is reduced to the linear differential ones

$$
\frac{d \sigma(t)}{d t}+\delta \sigma(t)=\sigma_{i} \gamma ; \delta=\gamma+\lambda ; \quad i=1,2
$$

The solution of the equation (27) is represented by the following function

$$
\sigma(t)=\sigma_{i}\left[\frac{\gamma}{\delta}+\frac{\lambda e^{-\delta(t-n T)}}{\delta}\right]
$$

where 


$$
\sigma_{i}= \begin{cases}\sigma_{1} ; & n=2 p \\ \sigma_{2} ; & n=2 p+1\end{cases}
$$

In [12] the noted problems are decided by the small parameter method. The stress $\sigma(t)$ is looked in the form

$$
\sigma(t)=\sum_{i=0}^{\infty} \xi^{i} \sigma_{i}(t)
$$

where $\xi$ is the small parameter and $\sigma(t)$ is the solution of corresponding linear integral equation. Evidently this approach reduces to a considerable calculs in applications.

\section{References}

1. W. Weibull, Trans. Roy. Inst. Technol.(Stockholm), 27 (1949)

2. L. Boltzmann, [WA-1, 616] (Wien. Ber. 70. S. 275-306. (1874) und Pogg. Ann. Erg.Bd. 7. S. 624 (1876))

3. V.M. Bondarenko, E.A. Larionov, Str. Mech. of Eng. Constr. and Build, 2 (2011)

4. E.A. Larionov, A.E. Larionov. Str. Mech. and Calculi build, 2 (2015)

5. N.T. Vasilkova, M.E. Bashcatova, E.A. Larionov, Str. Mech. of Eng. Constr. and Build,1 (2012)

6. E.A. Larionov, Vestnik MGSU, 4 (2016)

7. E.A. Larionov, Str. Mech. of Eng. Constr. and Build, 4 (2015)

8. O. Berg. The physical bases of strength theorg of concrete and reinforced concrete. (Stroyizdat, 1962)

9. A.A. Gvozdev. A calcul of bearing capacity of constructions by limit equilibrium method. (Stroyizdat, 1949)

10. Yu. N. Rabotnov. A creep of constructive elements, (Nauka, 1982)

11. A.A. Gvozdev, Mech. Solid, 5 (1972)

12. K.Z. Galustov. Non-linear creep theory of Concrete and calculation of reinforced constructions. (Fizmatlit, 2006)

13. V.M. Bondarenko, S.V. Bondarenko, Ingeneering methods of non-linear theory of reinforced concrete. (Stroyizdat, 1982)

14. R.S. Sanzharovskij. Str. Mech. of Eng. Constr. 1 (2014)

15. R.S. Sanzharovskij, M.M. Manchenko, Str. Mech. and Calculi build, 2 (2015)

16. R.S. Sanzharovskij, M.M. Manchenko, Str. Mech. of Eng. Constr. 3 (2016)

17. V.M. Bondarenko, Str. Mech. of Eng. Constr. 2 (2015) 